\title{
Review Article \\ Enhancing NMDA Receptor Function: Recent Progress on Allosteric Modulators
}

\author{
Lulu Yao and Qiang Zhou \\ School of Chemical Biology and Biotechnology, Peking University Shenzhen Graduate School, Shenzhen 518055, China \\ Correspondence should be addressed to Qiang Zhou; zhouqiang@pkusz.edu.cn
}

Received 5 August 2016; Revised 16 November 2016; Accepted 13 December 2016; Published 9 January 2017

Academic Editor: Cheng-Chang Lien

Copyright (C) 2017 L. Yao and Q. Zhou. This is an open access article distributed under the Creative Commons Attribution License, which permits unrestricted use, distribution, and reproduction in any medium, provided the original work is properly cited.

The N-methyl-D-aspartate receptors (NMDARs) are subtype glutamate receptors that play important roles in excitatory neurotransmission and synaptic plasticity. Their hypo- or hyperactivation are proposed to contribute to the genesis or progression of various brain diseases, including stroke, schizophrenia, depression, and Alzheimer's disease. Past efforts in targeting NMDARs for therapeutic intervention have largely been on inhibitors of NMDARs. In light of the discovery of NMDAR hypofunction in psychiatric disorders and perhaps Alzheimer's disease, efforts in boosting NMDAR activity/functions have surged in recent years. In this review, we will focus on enhancing NMDAR functions, especially on the recent progress in the generation of subunit-selective, allosteric positive modulators (PAMs) of NMDARs. We shall also discuss the usefulness of these newly developed NMDAR-PAMs.

\section{Introduction}

NMDARs belong to the L-glutamate family, and they play important roles in synaptic transmission, synaptic plasticity, and experience-dependent refinement of synaptic connections during development $[1,2]$. Their excessive activation or underactivation is proposed to contribute to the genesis or progression of various brain diseases, including stroke, schizophrenia, depression, and Alzheimer's disease $[1,3-5]$. Past efforts in targeting NMDARs for therapeutic intervention had been focused on inhibiting these receptors with only limited success [6-8].

NMDARs are regarded as coincidence detectors because of their ligand-gated and voltage-gated properties that its activation requires both binding of glutamate and coagonist (glycine or D-serine) and postsynaptic depolarization. In addition, NMDARs contain several regulatory sites sensitive to polyamines, $\mathrm{Zn}^{2+}$, protons, and glutathione $[1,9]$. The $\mathrm{X}$ ray crystal structure of the NMDAR shows massive protein complexes, and each complex is composed of four subunits, which contains amino-terminal domain participating in assembling and modulation; a transmembrane domain forming an ion-channel pore; c-terminal domain involving in the trafficking of receptors and coupling to intracellular signaling molecules; and a ligand-binding domain binding agonists [10, 11]. NMDARs are composed of subunits from seven homologous genes, GluN1, GluN2A-GluN2D, and GluN3A-GluN3B. NMDARs are diverse in subunit composition, biophysical, and pharmacological properties, interacting partners and subcellular localization. Among these subunits, the four GluN2 (A-D) subunits are major determinants of the functional heterogeneity of NMDARs [12]. Different spatiotemporal expression profile is also a prominent feature of NMDARs. GluN2B is the dominant subunit at early age and reaches its peak expression in the first postnatal week, while GluN2A is most abundant in the adult brain in rodents. During postnatal brain development, an activity-dependent switch from GluN2B to GluN2A occurs. Synaptic NMDARs mainly contain diheteromeric GluN1/GluN2A and triheteromeric GluN1/GluN2A/GluN2B NMDARs at excitatory synapses on excitatory neurons. The percentage of triheteromeric NMDARs are estimated between one-third and two-thirds of total NMDARs [13-16]. Perisynaptic and extrasynaptic sites are enriched in GluN2B-containing receptors which are considered by some to trigger excitotoxicity and cell death when excessively activated [9]. Different types of neurons may express somewhat different combination of NMDAR subunits. While GluN2A and GluN2B subunits are highly 
expressed in the excitatory neurons, GluN2C and GluN2D subunits are more concentrated in the inhibitory GABAergic neurons $[17,18]$.

\section{Enhancing NMDAR Functions}

2.1. The Need to Enhance NMADR Functions. Proper development and refinement of neural circuit require the adequate function/activity of NMDARs. This can be understood as NMDARs are required to support synaptic plasticity mostly on the excitatory neurons [19]. On the other hand, it has been increasingly recognized that NMDARs on the GABAergic inhibitory neurons contribute to second-by-second synaptic transmission and hence excitation of these inhibitory neurons. As a result, reduced function of NMDARs on these inhibitory neurons may hinder their physiological functions and lead to the imbalance between excitation and inhibition [20-27].

2.2. NMDAR's Role in the Certain CNS Diseases. Most NMDAR-targeting pharmacological agents that have been tested in the clinical trials are nonselective in that they do not distinguish between NMDAR subunits. These broad spectrum NMDAR inhibitors, such as dizocilpine (MK801), usually cause certain serious side effects including psychosis, memory impairment, and neuronal cell death. The majority of past efforts have been on generating inhibitors of NMDARs, for indications such as stroke, traumatic brain injury, and depression [29-31]. Ketamine has shown great promise in treating treatment-resistant depression with fast onset [32-34], although whether it is doing so via blocking NMDARs has been challenged recently [35]. Interestingly, rapastinel (also named GIYX-13) has shown antidepressant as an adjunctive therapy for treating depression [36]. GlYX13 acts as a selective, weak partial agonist of the glycine site on the NMDARs. Unlike ketamine, GLYX-13 does not elicit psychotomimetic side effects. Recent evidence showed that via modulating NMDARs GLYX-13 leads to an increase in mature dendritic spines and a persistent reduction in the threshold for future induction of LTP [37-39]. In addition, another likely more potent drug NMDAR enhancer, sarcosine, a glycine transporter-1 (GlyT-1) inhibitor, was shown to improve the depression-like behaviors and symptoms [40, 41].

It is noteworthy that the development of NMDAR blockers for stroke has been met with failure in clinical trials, likely because the elevation in glutamate concentration during stroke is short-lasting (about half an hour after stroke onset) and hence NMDARs do not have time to act (most stroke patients do not get treated for at least a few hours after stroke onset). In addition, inhibiting NMDAR activation is likely to hinder the recovery process after stroke [42].

Certain evidence showed that both mRNA and protein levels of NMDARs are reduced in AD brain and $\mathrm{AD}$ model, suggesting hypofunction of NMDAR in the pathogenesis of Alzheimer's disease [43]. However, the level of NMDARs at a given synapse is reduced with $\mathrm{AD}$ progression which has not been demonstrated directly. Shankar et al. suggested that $\mathrm{A} \beta$ oligomers decreased the density of dendritic spines, NMDAR-mediated calcium influx, and internalization of synaptic NMDARs $[44,45]$. Moreover, APP or ApoE4 mutant mice exhibit a decrease in NMDAR-mediated synaptic responses and impaired LTP; NMDAR hypofunction with advanced age is closely tied to redox state in the CNS [46]. However, in targeting NMDARs, only memantine has been approved by the FDA currently. Memantine is a NMDAR blocker and is thought to be neuroprotective by blocking excitotoxicity. It can be used for the treatment of moderate to severe $\mathrm{AD}$, and recent studies demonstrated that memantine shows benefits in improving cognition, behavior, and daily living in $\mathrm{AD}$ patients $[47,48]$.

Substantial pharmacological, genetic, and biochemical evidence show that NMDAR hypofunction may have key contributions to the genesis of schizophrenia. For example, NMDAR antagonists, such as ketamine and phencyclidine (PCP), can induce schizophrenia-like phenotypes (including positive, negative symptoms, and cognitive deficits) in healthy individuals and exacerbate such symptoms in schizophrenic patients [49-52]. Large genome wide association study of schizophrenia implicated numerous genes involved in glutamatergic transmission, such as genes encoding the GluN2A subunits and serine racemase [53, 54]. Postmortem brain samples from schizophrenic patients also suggested reduced NMDAR function. These observations support the on-going efforts to enhance the function/activity of NMDARs. When enhancing NMDAR function, caution has been taken to avoid excessive activation which likely leads to excitotoxicity. In this regard, targeting coagonist at the glycine binding site or using NMDAR-PAMs may be a safer therapeutics option [55].

\subsection{Glycine Binding Site Coagonists as NMDAR Enhancers.} Glycine binding site has attracted attention of many scientists as a potential target for safely elevating the activity of NMDARs. Glycine binding site activation is obligatory for NMDAR opening $[56,57]$. Glycine and D-serine are considered to be the two main endogenous coagonists. Functionally, glycine (or D-serine) binding increases the recovery rate for NMDARs and hence prolongs the duration of NMDAR EPSPs [58]. It can enhance the affinity and efficacy of glutamate binding on GluN2 subunit of NMDARs [59]. Electrophysiological results showed that both of the above actions can enhance NMDAR responses [60, 61]. Animal studies have shown that these coagonists and related modulators attenuated deficits in working memory and locomotor activity and reduced the deficits in prepulse inhibition [62, 63]. In recent years, a few clinical trials have shown efficacy of NMDAR enhancers in schizophrenia, such as DCS, sarcosine, and sodium benzoate. Participants receiving DCS showed enhanced potentiation of neural responses and cognitive performance $[64,65]$. Sodium benzoate, as a d-amino acid oxidase inhibitor, significantly improved the PANSS total score and neurocognition in chronic schizophrenia patients [66] (ClinicalTrials.gov NCT00960219.)

Although some clinical trials show that daily administration of a large quantity of exogenous glycine or D-serine alone, as well as partial coagonists D-cycloserine or using 
these coagonists as an adjunct to atypical antipsychotic treatment, could result in an improvement of cognitive function and attenuate negative symptoms and positive symptoms in schizophrenia patients $[3,67,68]$, other clinical trials had not produced positive outcomes; typically efficacy was seen in small trials while no efficacy was reported in larger trials [69, 70]. These observations suggest that NMDAR hypofunction may occur only in a subpopulation of schizophrenia patients, although how to identify this subpopulation is currently unknown. NMDAR modulators/enhancers are expected to be most effective in individuals exhibiting poor premorbid function, slow and incomplete response to antipsychotic agents, and relatively generalized nature of neurocognitive dysfunction [71]. The inconsistency between clinical trials using glycine may be explained by difference in the saturation of the glycine binding site, in that glycine will only be effective when the site is unsaturated. Whether the glycine binding site is saturated is still a matter of debate. Brain slice studies showed that the glycine site was saturated in the cerebellum but not in the PFC, hippocampus, and hypoglossal nucleus [72]. In vivo evidences also showed nonsaturation in the PFC $[73,74]$. How to resolve the inconsistent clinical trials and identify effective future therapy is a major challenge. Identifying biomarkers that are directly linked to or associated with the underlying pathology of schizophrenia will be a major step forward in the individualized diagnosis and therapy.

Among the reagents targeting the glycine binding site, glycine transporters (GlyTs) have attracted efforts from many researchers for their key roles in regulating glycine concentration in the vicinity of NMDARs $[75,76]$. Both GlyT-1 and GlyT-2 belong to the sodium-dependent solute carrier family 6. GlyT-1 and GlyT-2 show different regional and subcellular expression patterns. while GlyT-1 is expressed in most regions of the brain, mainly in glial cells and in presynaptic neurons, the expression of GlyT-2 is located on glycinergic neurons in brain stem and spinal cord [77-80]. Two GlyT-1 inhibitors have attracted a lot of attention. One is sarcosine, as mentioned above, an intermediate metabolite of glycine metabolism. It was shown to be beneficial for short-term treatment in acutely ill and chronically stable schizophrenia [81, 82], and in major depression [40]. The other GlyT-1 inhibitor, Bitopertin developed by HoffmannLa Roche, has been tested in clinical trials. Bitopertin reached phase III clinical testing for treating negative symptoms or positive symptoms in schizophrenia. These trials were halted due to lack of efficacy in improving negative symptoms, which is the primary endpoint for these trials [53]. However, in this year, a clinical trial in Japan population showed that Bitopertin can improve the "negative" and "suboptimally controlled" symptoms, and Bitopertin showed good safety and tolerated [83]. Bitopertin remains hopeful for treating schizophrenia.

Some studies reported that glycine primarily targeted the extrasynaptic NMDARs while D-serine regulates the activity of synaptic NMDARs [84]. If so, it suggests that elevating glycine may preferentially enhance the activation of extrasynaptic NMDARs which might not be sufficient or appropriate to modulate functions in schizophrenia patients.
Furthermore, Matsui et al. suggested that $\mathrm{D}$-serine was up to three times more potent than glycine on NMDA receptors [85]. Following this logic, augmenting synaptic NMDAR function by elevating D-serine level may be a more effective therapeutic option $[66,84]$.

Overall, targeting the glycine binding site enhances NMDAR-mediated response which may avoid excitoxicity and neuronal degeneration compared to targeting the glutamate binding site. In addition, reagents targeting the glycine binding site can readily cross the blood brain barrier which is convenient for clinical use. Many preclinical and clinical studies have shown that targeting the glycine binding site can be readily achieved, but certain issues need to be taken into consideration. First, long-term treatment with glycine binding site enhancer is required. Although acute efficacy of targeting glycine site has been reported in some clinical trials, there is no clear evidence for benefits after long-term treatment. On the other hand, there have been reports that chronic D-serine treatment promoted NMDAR internalization, chronic D-cycloserine treatment resulted in NMDAR desensitization [86], and chronic glycine treatment may preferentially act on extrasynaptic NMDARs [53]. Second, some studies suggested that excessive activation of glycine binding site may contribute to the excitotoxicity in neurodegenerative diseases, such as amyotrophic lateral sclerosis (ALS) [87]. If this is the case, this suggests a potential on-target toxicity which may prevent long-term dosing of such compounds. Third, some patients treated with glycine showed recurrent gastrointestinal upset [88]. Large doses of D-serine treatment may be associated with renal toxicity [89, 90], although clinical trials have not shown kidney dysfunction [91]. These observations suggest potential side effects associated with targeting the glycine binding site. Taken all together, although the rationale for targeting glycine binding site is clear and suitable drug-like candidates are available, this target may not be ideal for clinical applications, especially long-term.

2.4. NMDAR-PAMs. In addition to altering the level of coagonists (such as glycine) to enhance NMDAR activation, allosteric modulation is another option for enhancing NMDAR functions. Allosteric modulators have been regarded as the next generation CNS therapeutics based on the discovery and clinical success of benzodiazepines which enhance the $\mathrm{GABA}_{\mathrm{A}}$ receptors in an allosteric manner [92]. By definition, allosteric modulators have little or no effect in the absence of agonists (such as glutamate or GABA) but enhance (positive, PAMs) or reduce (negative, NAMs) the responses in the presence of agonists. This nature ensures that PAMs or NAMs only exert their actions physiologically at the right location (the active synapses) and at the right time (during receptor activation). Hence, PAMs/NAMs should have much reduced undesirable effects caused by excessive activation or inhibition. Typically, PAMs/NAMs do not bind at the agonist-binding sites of the targeted receptors. In addition, the saturability of allosteric modulators can decrease the risk of overdose, and PAMs/NAMs have high receptor selectivity since the binding sites of allosteric modulators are less conserved [6,93]. 
2.4.1. Early NMDAR-PAMs. Besides endogenous NMDARPAMs, such as histamine, ATP, spermine, $\mathrm{Mg}^{++}$, and neurosteroids, a few series of NMDAR-PAMs have been reported in the past years, including phenanthrene derivatives, naphthalene derivatives, and coumarin derivatives [94]. UBP compound is a family of agents targeting GluN1/GluN2 agonist-binding site of NMDARs. The subunit selectivity varies between compounds and either potentiation or inhibition has been observed. UBP512, a phenanthrene derivative, potentiated GluN2A-NMDARs with little or no effect on GluN2B-NMDARs and inhibited GluN1/GluN2C and GluN1/GluN2D NMDARs selectively. UBP551, a naphthalene derivative, potentiated GluN2D-NMDARs while inhibiting Glu2A-, Glu2B-, and Glu2C-NMADRs. The mechanism underlying the potentiation and inhibition is unknown [95, 96]. UBP714, a coumarin derivative, potentiated GluN2A-, GluN2B-, and GluN2D-NMDARs slightly [97]. CIQ, acting at the transmembrane domain, selectively potentiated GluN2Cor GluN2D-NMDARs [98, 99]. Chimeric receptor and point mutation studies demonstrated that the N-terminal domain, ligand-binding domain, and T592 of GluN2D played a key role in mediating the effect of CIQ [98]. However, whether these compounds are active or effective in a biological system has not been much tested.

2.4.2. The GNE Series of NMDAR-PAMs. Recently, a Genentech group has reported a few series of GluN2A-selective PAMs and has done fairly substantial characterizations on these PAMs, including structural analysis of binding sites and functional activity [28]. The rationale for generating subunit-selective, direct enhancer of NMDARs is to optimally enhance the physic activation of NMDARs while minimizing pathological activation. Some studies indicate that GluN2A-containing NMDARs are important for synaptic plasticity $[100,101]$, and their functions are reduced in certain brain diseases such as schizophrenia, while excessive activation of GluN2B-NMDARs may trigger excitotoxicity in such conditions as stroke/ischemia and Alzheimer's diseases [102-105]. Thus it might be desirable to generate GluN2Aselective NMDAR-PAMs which do not activate GluN2BNMDARs. These NMDAR-PAMs are generated first via a massive screening over 1.4 million compounds on GluN1GluN2A NMDAR expressing HEK293 cells, followed by medicinal chemistry effort to improve their selectivity and potency. The reported PAMs are in the uM potency range (EC50), with varying degree of selectivity over AMPARs and subunits of NMDARs.

The reported GNE NMDAR-PAMs are very selective against GluN2A, with little or no potentiation of GluN2B, and with about 10-fold lower potency towards GluN2D. Since, in the adult, GluN2D is mainly expressed on the inhibitory neurons in the midbrain [106], the nonselective effects on these neurons have been limited on the general brain function. Most of the reported PAMs are also highly selective against the AMPARs. There is certain evidence that, in addition to diheteromeric form, triheteromeric form represents a significant fraction of total NMDARs and contributes to synaptic function and plasticity [14, 107]. These triheteromeric NMDARs are also potentiated by PAMs, although not as effective as the diheteromeric ones. The selectivity of PAMs against GluN2A-NMDARs was confirmed by a lack of effect in the GluN2A-KO mice.

How is this subunit selectivity achieved? Analysis of crystal structure of the dimer of GluN1-GluN2A ligandbinding domain in complex with GNE-6901 revealed that it binds to this region of the NMDARs. Further analysis identified V783 in GluN2A as a critical residue to endow the subunit selectivity of GNE NMDAR-PAMs. While V783 engages GNE-6901 via direct van der Waals contact, the corresponding residue F784 in GluN2B is much larger, protrudes to the binding sites, and may prevent GNE-6901 from binding to GluN2B-NMDARs. For the AMPARs, it appears that GNE-6901 acts as a silent allosteric modulator in that it can bind to the analogous site in the AMPARs but does not have any efficacy at this site. Thus, the selectivity against AMPARs is achieved via absence of efficacy rather than potency.

There are a few mechanisms that NMDAR-PAMs can potentiate the agonist-induced responses. One mechanism is by enhancing the potency of agonists which is exhibited as a leftward shift on the agonist concentration-response curve; that is, the same magnitude of responses can be obtained at lower agonist concentrations in the presence of PAMs. This situation may be especially useful or effective when the agonist is not saturated. Since NMDARs have two coagonists, glutamate and glycine, this increase in potency could be mediated by enhanced potency of glutamate, glycine, or both. GNE-8324 caused a significant increase in glutamate potency while GNE-6901 had much smaller effect; neither affected glycine potency. Another mechanism of PAMs is to prolong the activation of receptors, such as by reducing deactivation. Both GNE-6901 and GNE-8324 showed slowing of kinetics at saturating concentration of glutamate which is due to reduced deactivation, with GNE-8324 more pronounced than GNE6901. Specifically, the potentiation by GNE-8324's effect had a much bigger dependence on the glutamate concentration than GNE-6901.

A very interesting and important result of this study is that Hackos et al. had identified two types of NMDAR-PAMs [28], GNE-6901 that potentiated NMDARs on both excitatory and inhibitory neurons and GNE-8324 that only potentiated NMDARs on the inhibitory neurons (Figure 1). The mechanism underlying this differential potentiation is still unclear at this moment. One possibility is that the synaptic glutamate concentrations at the glutamatergic synapses on inhibitory neurons are much larger than that on the excitatory neurons. This interesting feature of GNE-8324 allows it to be used as a great tool to examine the effects of selectively enhancing inhibition (see below).

NMDARs are required for the induction of long-term potentiation (LTP) of glutamatergic synapses on the excitatory neurons, and hence the PAMs of NMDAR are supposed to enhance LTP. This is certainly the case for GNE-6901, but not for GNE-8324 (Figure 2). To further understand how much effect the PAMs have on the activation of NMDARs during LTP induction (with theta burst stimulation, TBS), NMDAR responses from the pyramidal neurons during TBS were calculated from recordings. Interestingly, the magnitude 


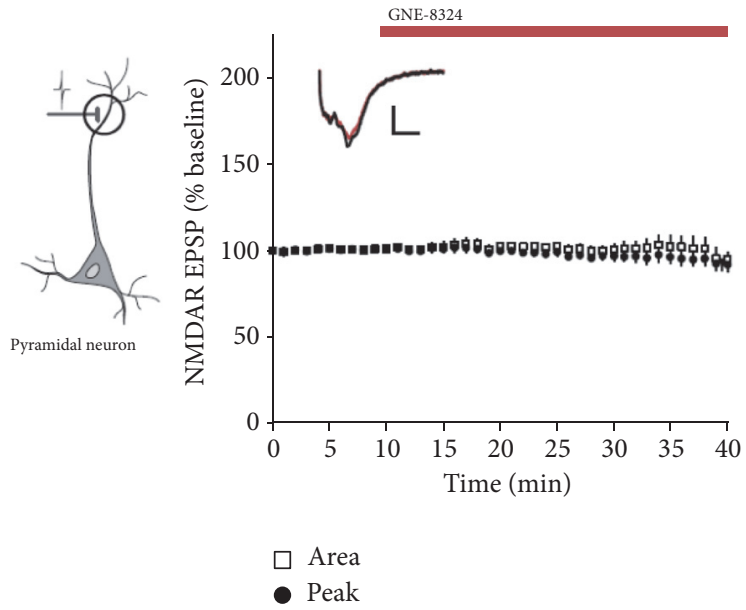

(a)

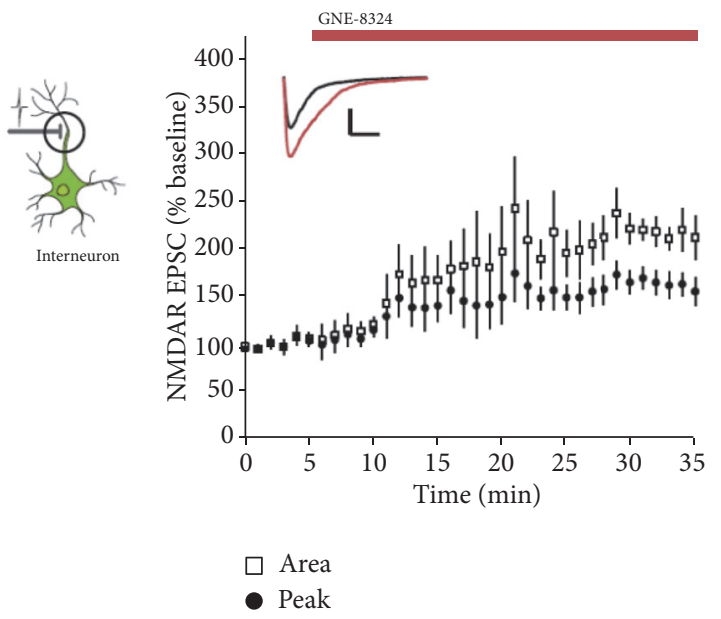

(c)

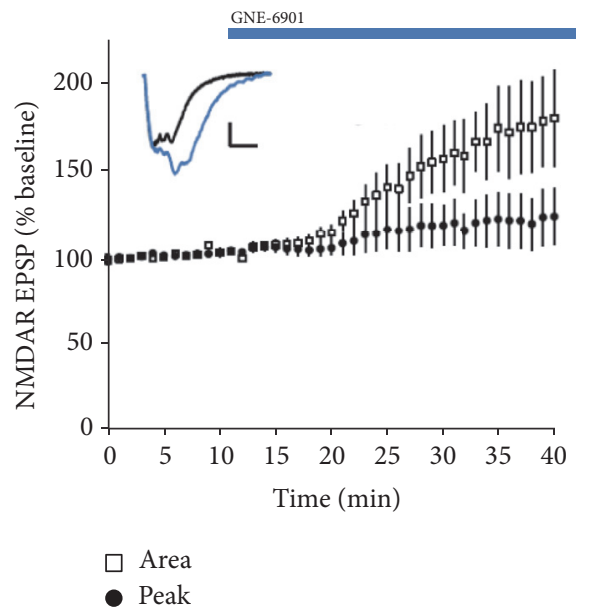

(b)

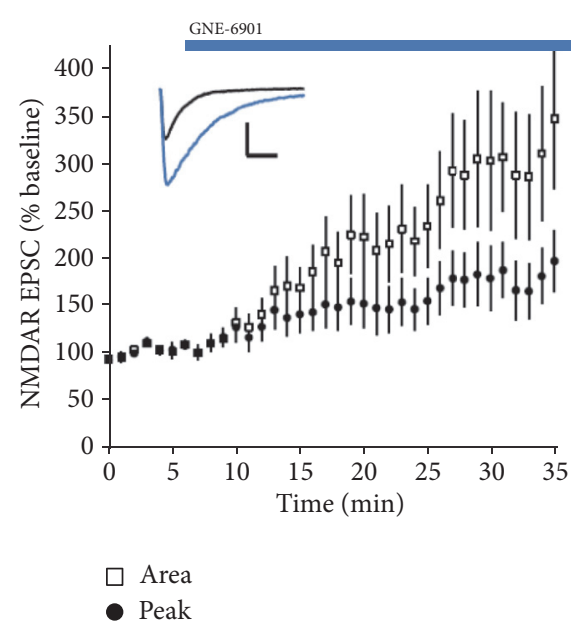

(d)

FIgURE 1: Cell type-specific effects of NMDAR-PAMs. (a) GNE-8324 has no effect on NMDAR EPSPs recorded from CA1 pyramidal neurons in acute brain slices. (b) In contrast, GNE-6901 showed robust potentiation on NMDAR EPSPs under the same condition. (c, d) Robust potentiation of both GNE-8324 and GNE-6901 on NMDAR EPSCs recorded in the inhibitory neurons in the hippocampus. Modified from [28].

of enhancement during TBS depended on whether inhibition was intact. When inhibition was intact (as seen under physiological conditions), a significant enhancement of NMDAR responses by GNE-6901 was seen; but for GNE-8324 there was a small but significant reduction. These results can certainly explain the differential effects of PAMs on LTP. On the other hand, when inhibition was blocked, the reduction in NMDAR responses by GNE-8324 was switched to a small and nonsignificant enhancement, indicating that activation of NMDARs on the inhibitory neurons is responsible for the reduced NMDAR responses on excitatory neurons during TBS. Smaller LTP may be beneficial in certain diseases, such as schizophrenia. An enhanced LTP has been reported in a mouse model of schizophrenia, which is likely caused by reduced inhibition [108]. Recently, Volgraf et al. have reported the synthesis of potent GluN2A-selective NMDARs PAMs, such as GNE-0723, which is highly brain penetrant. In addition to extensively testing in brain slices and on oocytes,
GNE-0723 has been shown to be suitable for in vivo testing of GluN2A-PAMs [109]. The future in vivo studies on NMDARPAMs will provide more definitive testing for their potential in drug development.

2.4.3. Important Unresolved Issues. Although the GNE series of NMDAR-PAMs have provided many new insights into the functioning of NMDARs and their contributions to brain under both physiological and pathological conditions, we have only seen the tip of the iceberg. Here, we have listed three important issues that can be resolved by using these PAMs as tool compounds.

(1) Excitoxicity: Is It Subunit-Dependent? It is still debated whether excitotoxicity is directly related to or caused by the subunit composition or the subcellular locations of the NMDARs that are excessively activated during pathological conditions such as stroke. In such context, it has 

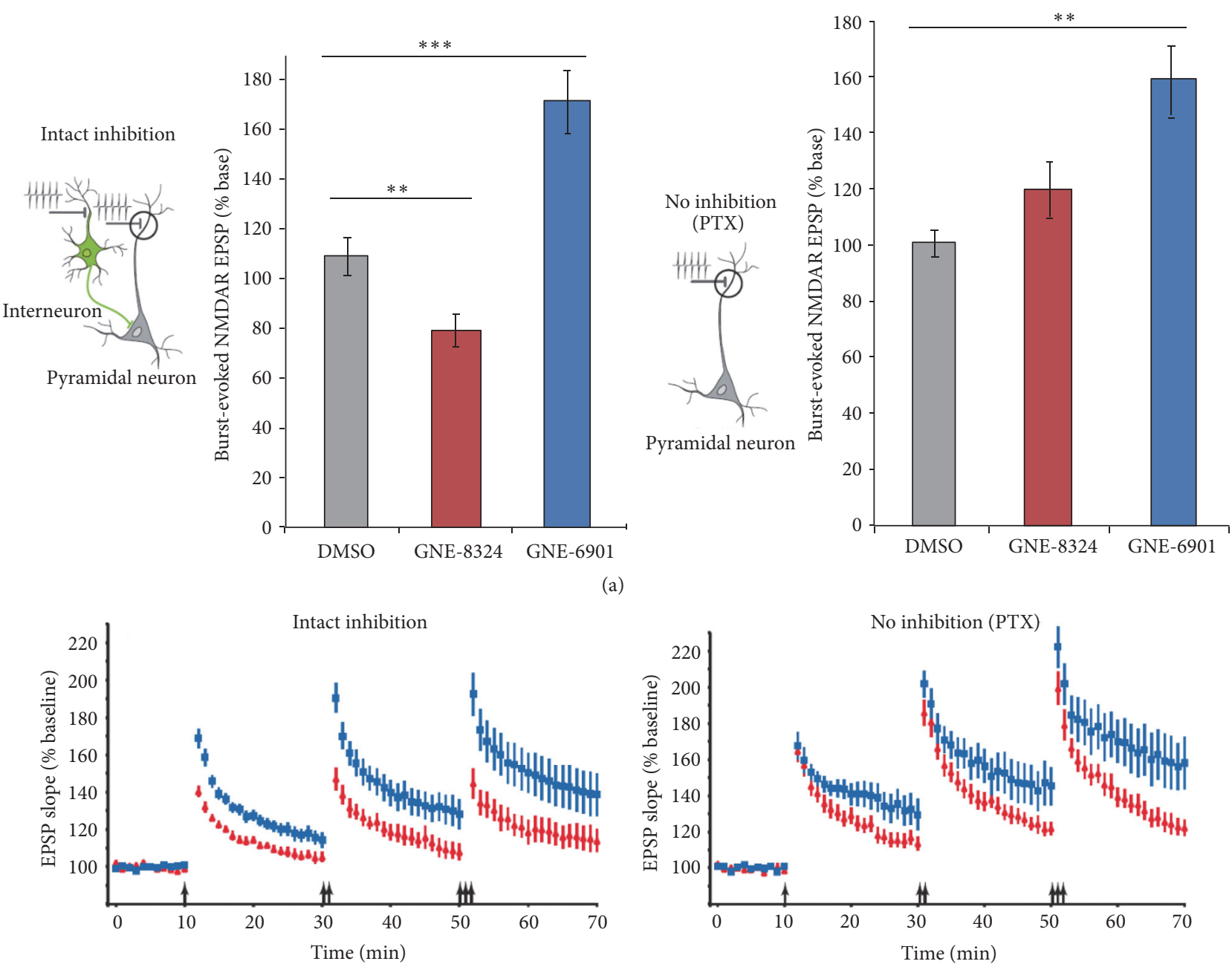

GNE-6901

- GNE-6901

$\Delta$ GNE-8324

$\Delta$ GNE-8324

(b)

Figure 2: Differential effects on NMDARs underlie different modification of synaptic plasticity by GNE-PAMs. (a) NMDAR-mediated responses during TBS were calculated and showed differences between GNE-8324 and GNE-6901 which further depends on whether inhibition is present. With intact inhibition, GNE-6901 enhanced while GNE-8324 reduced NMDAR responses, compared to controls. When inhibition was absent, GNE-8324 showed a small, nonsignificant enhancement. (b) Consistent with the above effects on NMDAR responses, GNE-6901 enhanced LTP regardless of whether inhibition was present, while GNE-8324 enhanced LTP in the absence of inhibition but reduced LTP when inhibition was intact. Modified from [28].

been proposed that either GluN2B-NMDARs or extrasynaptic NMDARs are responsible for inducing excitotoxicity $[102,103,110,111]$. If GluN2A-selective PAMs can enhance NMDARs that do not contain GluN2B subunits in the absence of causing much excitotoxicity, then it can be argued that the subunit model is likely correct. However, it is not understood whether enhancing activation of physiological activity itself is sufficient to trigger excitotoxicity. In this regard, a control experiment using NMDAR-PAMs that can selectively enhance GluN2B-NMDARs will be critical. Thus, NMDAR-PAMs can be used as a tool compound to test the contribution of subunit composition in excitotoxicity.
(2) Kinetics: How Much Does It Matter? Although the ideal situation is that enhanced activation preserves the kinetics of the receptor response, the nature/mechanism of the current NMDAR-PAMs dictates that this wish may not easily be granted. When enhancement involves reduced deactivation and hence slowing of kinetics, the responses mediated by activation of NMDARs may affect the encoding and transmission of information. For example, if potentiation of NMDAR response is sufficient to trigger more spiking activity in neurons due to prolonged depolarization, the increased numbers of spikes per given input may alter the encoding of sensory information or degrade their temporal patterns. This scenario 
may not be a major issue for NMDARs on the excitatory neurons since their major role is enabling the induction of synaptic plasticity (which is mostly a threshold-triggering event) compared to the second-by-second transmission of information. However, for inhibitory neurons, things might be different since there is ample evidence that NMDARs on these neurons are involved in the integration of inputs and generation of spiking activity [112], although there is no direct test of whether the dynamics of NMDARs affect processing or synaptic transmission in these inhibitory neurons in vivo. Some researchers looked at the contribution of NMADRs to the functioning of fast-spiking interneurons in the above context and they concluded that there is very limited contribution since the density of NMDARs on these neurons is very low [112-116]. Again, these new GNE NMDAR-PAMs may provide a great opportunity to understand this issue and the best test is to compare information encoding and synaptic transmission in vivo in the absence and presence of NMDARPAMs.

(3) Enhancing Inhibition: Is It Sufficient? Quite a bit emphasis has been put on the contribution of inhibitory neurons to the genesis of various CNS diseases, such as schizophrenia and Alzheimer's disease [1]. What these studies appear to suggest is that inhibitory neurons are directly affected (such as having reduced synapse density or spiking) rather than the postsynaptic GABA receptors, at least at the early stage of the diseases. Thus, a direct test to this hypothesis, as well as a potential new way to treat such diseases, is to enhance inhibitory functions by enhancing the activity of the affected inhibitory neurons. So far this idea has not been tested due to the lack of reagents to directly and selectively enhancing the activity in the inhibitory neurons. Since GNE-8324 can selectively enhance the NMADRs on the inhibitory neurons, it can be used to test the above hypothesis and may thus provide some new insights into the contribution of inhibition to the genesis of certain brain diseases and the potential of enhancing inhibition as therapeutics.

\section{Prospective and Future Research}

From selectively inhibiting NMDARs to enhancing their functions, we have undergone a drastic change in our thinking about the nature and contribution of these important receptors to physiological functions and pathological processes. Although there have been a lot of excitements with the recent rapid progress in the generation of various series of distinct NMDAR-PAMs, we are only at the very beginning of a new era to use these compounds as tools to further understand the nature of NMADRs, brain functions, and whether enhancing NMDARs can be a viable option in treating brain diseases.

\section{Competing Interests}

The authors declare that they have no competing interests.

\section{Acknowledgments}

This work was supported by grants from Peking University Shenzhen Graduate School and grants from Shenzhen government (ZDSYS201504301539161 and KQTD2015032709315529).

\section{References}

[1] P. Paoletti, C. Bellone, and Q. Zhou, "NMDA receptor subunit diversity: impact on receptor properties, synaptic plasticity and disease," Nature Reviews Neuroscience, vol. 14, no. 6, pp. 383400, 2013.

[2] S. F. Traynelis, L. P. Wollmuth, C. J. McBain et al., "Glutamate receptor ion channels: structure, regulation, and function," Pharmacological Reviews, vol. 62, no. 3, pp. 405-496, 2010.

[3] C. R. Yang and K. A. Svensson, "Allosteric modulation of NMDA receptor via elevation of brain glycine and d-serine: the therapeutic potentials for schizophrenia," Pharmacology and Therapeutics, vol. 120, no. 3, pp. 317-332, 2008.

[4] C. G. Lau and R. S. Zukin, "NMDA receptor trafficking in synaptic plasticity and neuropsychiatric disorders," Nature Reviews Neuroscience, vol. 8, no. 6, pp. 413-426, 2007.

[5] Q. Zhou and M. Sheng, "NMDA receptors in nervous system diseases," Neuropharmacology, vol. 74, pp. 69-75, 2013.

[6] S. Zhu and P. Paoletti, "Allosteric modulators of NMDA receptors: multiple sites and mechanisms," Current Opinion in Pharmacology, vol. 20, pp. 14-23, 2015.

[7] V. E. O'Collins, M. R. Macleod, G. A. Donnan, L. L. Horky, B. H. Van Der Worp, and D. W. Howells, "1,026 Experimental treatments in acute stroke," Annals of Neurology, vol. 59, no. 3, pp. 467-477, 2006.

[8] L. V. Kalia, S. K. Kalia, and M. W. Salter, "NMDA receptors in clinical neurology: excitatory times ahead," The Lancet Neurology, vol. 7, no. 8, pp. 742-755, 2008.

[9] S. Cull-Candy, S. Brickley, and M. Farrant, "NMDA receptor subunits: diversity, development and disease," Current Opinion in Neurobiology, vol. 11, no. 3, pp. 327-335, 2001.

[10] E. Karakas and H. Furukawa, "Crystal structure of a heterotetrameric NMDA receptor ion channel," Science, vol. 344, no. 6187, pp. 992-997, 2014.

[11] C.-H. Lee, W. Lü, J. C. Michel et al., "NMDA receptor structures reveal subunit arrangement and pore architecture," Nature, vol. 511, no. 7508, pp. 191-197, 2014.

[12] H. Yuan, K. B. Hansen, K. M. Vance, K. K. Ogden, and S. F. Traynelis, "Control of NMDA receptor function by the NR2 subunit amino-terminal domain," Journal of Neuroscience, vol. 29, no. 39, pp. 12045-12058, 2009.

[13] K. B. Hansen, K. K. Ogden, H. Yuan, and S. F. Traynelis, "Distinct functional and pharmacological properties of triheteromeric GluN1/GluN2A/GluN2B NMDA receptors," Neuron, vol. 81, no. 5, pp. 1084-1096, 2014.

[14] K. R. Tovar, M. J. McGinley, and G. L. Westbrook, "Triheteromeric NMDA receptors at hippocampal synapses," The Journal of Neuroscience, vol. 33, no. 21, pp. 9150-9160, 2013.

[15] C. J. Hatton and P. Paoletti, "Modulation of triheteromeric NMDA receptors by N-terminal domain ligands," Neuron, vol. 46, no. 2, pp. 261-274, 2005.

[16] R. A. Al-Hallaq, T. P. Conrads, T. D. Veenstra, and R. J. Wenthold, "NMDA di-heteromeric receptor populations and 
associated proteins in rat hippocampus," Journal of Neuroscience, vol. 27, no. 31, pp. 8334-8343, 2007.

[17] I. Riebe, H. Seth, G. Culley et al., "Tonically active NMDA receptors-a signalling mechanism critical for interneuronal excitability in the CA1 stratum radiatum," European Journal of Neuroscience, vol. 43, no. 2, pp. 169-178, 2016.

[18] J. von Engelhardt, C. Bocklisch, L. Tönges, A. Herb, M. Mishina, and H. Monyer, "GluN2D-containing NMDA receptorsmediate synaptic currents in hippocampal interneurons and pyramidal cells in juvenile mice," Frontiers in Cellular Neuroscience, vol. 9, article 95, 2015.

[19] G. L. Collingridge, S. Peineau, J. G. Howland, and Y. T. Wang, "Long-term depression in the CNS," Nature Reviews Neuroscience, vol. 11, no. 7, pp. 459-473, 2010.

[20] S. M. Cohen, R. W. Tsien, D. C. Goff, and M. M. Halassa, "The impact of NMDA receptor hypofunction on GABAergic neurons in the pathophysiology of schizophrenia," Schizophrenia Research, vol. 167, no. 1-3, pp. 98-107, 2015.

[21] J. E. Belforte, V. Zsiros, E. R. Sklar et al., "Postnatal NMDA receptor ablation in corticolimbic interneurons confers schizophrenia-like phenotypes," Nature Neuroscience, vol. 13, no. 1, pp. 76-83, 2010.

[22] K. Nakazawa, V. Zsiros, Z. Jiang et al., "GABAergic interneuron origin of schizophrenia pathophysiology," Neuropharmacology, vol. 62, no. 3, pp. 1574-1583, 2012.

[23] D. A. Lewis and B. Moghaddam, "Cognitive dysfunction in schizophrenia: convergence of $\gamma$-aminobutyric acid and glutamate alterations," Archives of Neurology, vol. 63, no. 10, pp. 13721376, 2006.

[24] M. Carlén, K. Meletis, J. H. Siegle et al., "A critical role for NMDA receptors in parvalbumin interneurons for gamma rhythm induction and behavior," Molecular Psychiatry, vol. 17, no. 5, pp. 537-548, 2012.

[25] X. Gu, L. Zhou, and W. Lu, "An NMDA receptor-dependent mechanism underlies inhibitory synapse development," Cell Reports, vol. 14, no. 3, pp. 471-478, 2016.

[26] T.-U. W. Woo, J. P. Walsh, and F. M. Benes, "Density of glutamic acid decarboxylase 67 messenger RNA-containing neurons that express the N-methyl-D-aspartate receptor subunit NR2A in the anterior cingulate cortex in schizophrenia and bipolar disorder," Archives of General Psychiatry, vol. 61, no. 7, pp. 649657, 2004.

[27] D. A. Lewis, T. Hashimoto, and D. W. Volk, "Cortical inhibitory neurons and schizophrenia," Nature Reviews Neuroscience, vol. 6, no. 4, pp. 312-324, 2005.

[28] D. H. Hackos, P. J. Lupardus, T. Grand et al., "Positive allosteric modulators of GluN2A-containing NMDARs with distinct modes of action and impacts on circuit function," Neuron, vol. 89, no. 5, pp. 983-999, 2016.

[29] D. Catarzi, V. Colotta, and F. Varano, "Competitive Gly/NMDA receptor antagonists," Current Topics in Medicinal Chemistry, vol. 6, no. 8, pp. 809-821, 2006.

[30] K. W. Muir, "Glutamate-based therapeutic approaches: clinical trials with NMDA antagonists," Current Opinion in Pharmacology, vol. 6, no. 1, pp. 53-60, 2006.

[31] C. G. Abdallah, G. Sanacora, R. S. Duman, and J. H. Krystal, "Ketamine and rapid-acting antidepressants: a window into a new neurobiology for mood disorder therapeutics," Annual Review of Medicine, vol. 66, pp. 509-523, 2015.

[32] R. M. Berman, A. Cappiello, A. Anand et al., "Antidepressant effects of ketamine in depressed patients," Biological Psychiatry, vol. 47, no. 4, pp. 351-354, 2000.
[33] J. W. Murrough, A. M. Perez, S. Pillemer et al., "Rapid and longer-term antidepressant effects of repeated ketamine infusions in treatment-resistant major depression," Biological Psychiatry, vol. 74, no. 4, pp. 250-256, 2013.

[34] R. S. Duman, G. K. Aghajanian, G. Sanacora, and J. H. Krystal, "Synaptic plasticity and depression: new insights from stress and rapid-acting antidepressants," Nature Medicine, vol. 22, no. 3, pp. 238-249, 2016.

[35] P. Zanos, R. Moaddel, P. J. Morris et al., "NMDAR inhibitionindependent antidepressant actions of ketamine metabolites," Nature, vol. 533, no. 7604, pp. 481-486, 2016.

[36] S. Preskorn, M. Macaluso, V. Mehra, G. Zammit, J. R. Moskal, and R. M. Burch, "Randomized proof of concept trial of GLYX13 , an N-methyl-D-aspartate receptor glycine site partial agonist, in major depressive disorder nonresponsive to a previous antidepressant agent," Journal of Psychiatric Practice, vol. 21, no. 2, pp. 140-149, 2015.

[37] J. Burgdorf, X.-L. Zhang, C. Weiss et al., "The long-lasting antidepressant effects of rapastinel (GLYX-13) are associated with a metaplasticity process in the medial prefrontal cortex and hippocampus," Neuroscience, vol. 308, pp. 202-211, 2015.

[38] J. Burgdorf, R. A. Kroes, X.-L. Zhang et al., "Rapastinel (GLYX13) has therapeutic potential for the treatment of post-traumatic stress disorder: characterization of a NMDA receptor-mediated metaplasticity process in the medial prefrontal cortex of rats," Behavioural Brain Research, vol. 294, pp. 177-185, 2015.

[39] J. R. Moskal, J. S. Burgdorf, P. K. Stanton et al., "The Development of Rapastinel (Formerly GLYX-13); A rapid acting and long lasting antidepressant," Current Neuropharmacology, vol. 15, no. 1, pp. 47-56, 2016.

[40] C.-C. Huang, I.-H. Wei, C.-L. Huang et al., "Inhibition of glycine transporter-I as a novel mechanism for the treatment of depression," Biological Psychiatry, vol. 74, no. 10, pp. 734-741, 2013.

[41] K. Chen, C. Wu, M. Tsai et al., "Antidepressant-like effects of long-term sarcosine treatment in rats with or without chronic unpredictable stress," Behavioural Brain Research, vol. 316, pp. 1-10, 2017.

[42] T. W. Lai, W.-C. Shyu, and Y. T. Wang, "Stroke intervention pathways: NMDA receptors and beyond," Trends in Molecular Medicine, vol. 17, no. 5, pp. 266-275, 2011.

[43] A. J. Mishizen-Eberz, R. A. Rissman, T. L. Carter, M. D. Ikonomovic, B. B. Wolfe, and D. M. Armstrong, "Biochemical and molecular studies of NMDA receptor subunits NR1/2A/2B in hippocampal subregions throughout progression of Alzheimer's disease pathology," Neurobiology of Disease, vol. 15, no. 1, pp. 80-92, 2004.

[44] G. M. Shankar, B. L. Bloodgood, M. Townsend, D. M. Walsh, D. J. Selkoe, and B. L. Sabatini, "Natural oligomers of the Alzheimer amyloid- $\beta$ protein induce reversible synapse loss by modulating an NMDA-type glutamate receptor-dependent signaling pathway," Journal of Neuroscience, vol. 27, no. 11, pp. 2866-2875, 2007.

[45] E. M. Snyder, Y. Nong, C. G. Almeida et al., "Regulation of NMDA receptor trafficking by amyloid- $\beta$," Nature Neuroscience, vol. 8, no. 8, pp. 1051-1058, 2005.

[46] M. Guidi, A. Kumar, and T. C. Foster, "Impaired attention and synaptic senescence of the prefrontal cortex involves redox regulation of NMDA receptors," Journal of Neuroscience, vol. 35, no. 9, pp. 3966-3977, 2015. 
[47] J. Jiang and H. Jiang, "Efficacy and adverse effects of memantine treatment for Alzheimer's disease from randomized controlled trials," Neurological Sciences, vol. 36, no. 9, pp. 1633-1641, 2015.

[48] M. Knapp, D. King, R. Romeo et al., "Cost-effectiveness of donepezil and memantine in moderate to severe Alzheimer's disease (the DOMINO-AD trial)," International Journal of Geriatric Psychiatry, 2016.

[49] J. W. Olney and N. B. Farber, "Glutamate receptor dysfunction and schizophrenia," Archives of General Psychiatry, vol. 52, no. 12, pp. 998-1007, 1995.

[50] J. T. Coyle, A. Basu, M. Benneyworth, D. Balu, and G. Konopaske, "Glutamatergic synaptic dysregulation in schizophrenia: therapeutic implications," Handbook of Experimental Pharmacology, vol. 213, pp. 267-295, 2012.

[51] A. Parwani, M. A. Weiler, T. A. Blaxton et al., "The effects of a subanesthetic dose of ketamine on verbal memory in normal volunteers," Psychopharmacology, vol. 183, no. 3, pp. 265-274, 2005.

[52] G. Gilmour, S. Dix, L. Fellini et al., "NMDA receptors, cognition and schizophrenia-testing the validity of the NMDA receptor hypofunction hypothesis," Neuropharmacology, vol. 62, no. 3, pp. 1401-1412, 2012.

[53] D. T. Balu and J. T. Coyle, "The NMDA receptor 'glycine modulatory site' in schizophrenia: D-serine, glycine, and beyond," Current Opinion in Pharmacology, vol. 20, pp. 109-115, 2015.

[54] S. Ripke, B. M. Neale, A. Corvin et al., "Biological insights from 108 schizophrenia-associated genetic loci," Nature, vol. 511, no. 7510, pp. 421-427, 2014.

[55] D. R. Lynch and R. P. Guttmann, "Excitotoxicity: perspectives based on N-methyl-D-aspartate receptor subtypes," Journal of Pharmacology and Experimental Therapeutics, vol. 300, no. 3, pp. 717-723, 2002.

[56] M. C. Currás and B. S. Pallotta, "Single-channel evidence for glycine and NMDA requirement in NMDA receptor activation," Brain Research, vol. 740, no. 1-2, pp. 27-40, 1996.

[57] R. A. J. Lester, G. Tong, and C. E. Jahr, "Interactions between the glycine and glutamate binding sites of the NMDA receptor," Journal of Neuroscience, vol. 13, no. 3, pp. 1088-1096, 1993.

[58] L. Vyklický Jr., M. Benveniste, and M. L. Mayer, "Modulation of N-methyl-D-aspartic acid receptor desensitization by glycine in mouse cultured hippocampal neurones," The Journal of Physiology, vol. 428, no. 1, pp. 313-331, 1990.

[59] E. Fadda, W. Danysz, J. T. Wroblewski, and E. Costa, "Glycine and $\mathrm{D}$-serine increase the affinity of $\mathrm{N}$-methyl-D-aspartate sensitive glutamate binding sites in rat brain synaptic membranes," Neuropharmacology, vol. 27, no. 11, pp. 1183-1185, 1988.

[60] J. W. Johnson and P. Ascher, "Glycine potentiates the NMDA response in cultured mouse brain neurons," Nature, vol. 325, no. 6104, pp. 529-531, 1987.

[61] J.-P. Mothet, A. T. Parent, H. Wolosker et al., "D-serine is an endogenous ligand for the glycine site of the N-methylD- aspartate receptor," Proceedings of the National Academy of Sciences of the United States of America, vol. 97, no. 9, pp. 49264931, 2000.

[62] N. Kanahara, E. Shimizu, S. Ohgake et al., "Glycine and Dserine, but not $\mathrm{d}$-cycloserine, attenuate prepulse inhibition deficits induced by NMDA receptor antagonist MK-801," Psychopharmacology, vol. 198, no. 3, pp. 363-374, 2008.

[63] H. Sershen, A. Hashim, D. S. Dunlop, R. F. Suckow, T. B. Cooper, and D. C. Javitt, "Modulating NMDA receptor function with d-amino acid oxidase inhibitors: understanding functional activity in PCP-treated mouse model," Neurochemical Research, vol. 41, no. 1-2, pp. 398-408, 2016.

[64] J. K. Forsyth, P. Bachman, D. H. Mathalon, B. J. Roach, R. F. Asarnow, and K. Foerde, "Augmenting NMDA receptor signaling boosts experience-dependent neuroplasticity in the adult human brain," Proceedings of the National Academy of Sciences of the United States of America, vol. 112, no. 50, pp. 15331-15336, 2015.

[65] J. T. Kantrowitz, S. W. Woods, E. Petkova et al., "D-serine for the treatment of negative symptoms in individuals at clinical high risk of schizophrenia: a pilot, double-blind, placebo-controlled, randomised parallel group mechanistic proof-of-concept trial," The Lancet Psychiatry, vol. 2, no. 5, pp. 403-412, 2015.

[66] H.-Y. Lane, C.-H. Lin, M. F. Green et al., "Add-on treatment of benzoate for schizophrenia: a randomized, double-blind, placebo-controlled trial of D-amino acid oxidase inhibitor," JAMA Psychiatry, vol. 70, no. 12, pp. 1267-1275, 2013.

[67] D. Goff, "D-cycloserine in schizophrenia: new strategies for improving clinical outcomes by enhancing plasticity," Current Neuropharmacology, vol. 15, no. 1, pp. 21-34, 2016.

[68] G. E. Tsai and P.-Y. Lin, "Strategies to enhance N-Methyl-DAspartate receptor-mediated neurotransmission in schizophrenia, a critical review and meta-analysis," Current Pharmaceutical Design, vol. 16, no. 5, pp. 522-537, 2010.

[69] R. W. Buchanan, D. C. Javitt, S. R. Marder et al., "The Cognitive and Negative Symptoms in Schizophrenia Trial (CONSIST): the efficacy of glutamatergic agents for negative symptoms and cognitive impairments," The American Journal of Psychiatry, vol. 164, no. 10, pp. 1593-1602, 2007.

[70] H.-Y. Lane, Y.-C. Chang, Y.-C. Liu, C.-C. Chiu, and G. E. Tsai, "Sarcosine or D-serine add-on treatment for acute exacerbation of schizophrenia: a randomized, double-blind, placebocontrolled study," Archives of General Psychiatry, vol. 62, no. 11, pp. 1196-1204, 2005.

[71] M. A. Geyer and G. Gross, Eds., Novel Antischizophrenia Treatments, vol. 213, Springer, Berlin, Germany, 2012.

[72] D. Billups and D. Attwell, "Active release of glycine or Dserine saturates the glycine site of NMDA receptors at the cerebellar mossy fibre to granule cell synapse," European Journal of Neuroscience, vol. 18, no. 11, pp. 2975-2980, 2003.

[73] L. Chen, M. Muhlhauser, and C. R. Yang, "Glycine tranporter1 blockade potentiates NMDA-mediated responses in rat prefrontal cortical neurons in vitro and in vivo," Journal of Neurophysiology, vol. 89, no. 2, pp. 691-703, 2003.

[74] V. Labrie and J. C. Roder, "The involvement of the NMDA receptor d-serine/glycine site in the pathophysiology and treatment of schizophrenia," Neuroscience and Biobehavioral Reviews, vol. 34, no. 3, pp. 351-372, 2010.

[75] G. G. Kinney, C. Sur, M. Burno et al., "The glycine transporter type 1 inhibitor $\mathrm{N}$-[3-(4/-fluorophenyl)-3-(4/phenylphenoxy)propyl] sarcosine potentiates NMDA receptormediated responses in vivo and produces an antipsychotic profile in rodent behavior," Journal of Neuroscience, vol. 23, no. 20, pp. 7586-7591, 2003.

[76] R. J. Harvey and B. K. Yee, "Glycine transporters as novel therapeutic targets in schizophrenia, alcohol dependence and pain," Nature Reviews Drug Discovery, vol. 12, no. 11, pp. 866885, 2013.

[77] C. Romei and L. Raiteri, "Advances in understanding the functions of native GlyT1 and GlyT2 neuronal glycine transporters," Neurochemistry International, vol. 99, pp. 169-177, 2016. 
[78] B. Cubelos, C. Giménez, and F. Zafra, "Localization of the GLYT1 glycine transporter at glutamatergic synapses in the rat brain," Cerebral Cortex, vol. 15, no. 4, pp. 448-459, 2005.

[79] P. Legendre, "The glycinergic inhibitory synapse," Cellular and Molecular Life Sciences, vol. 58, no. 5-6, pp. 760-793, 2001.

[80] K. E. Smith, L. A. Borden, P. R. Hartig, T. Branchek, and R. L. Weinshank, "Cloning and expression of a glycine transporter reveal colocalization with NMDA receptors," Neuron, vol. 8, no. 5, pp. 927-935, 1992.

[81] H.-Y. Lane, Y.-C. Liu, C.-L. Huang et al., "Sarcosine (NMethylglycine) treatment for acute schizophrenia: a randomized, double-blind study," Biological Psychiatry, vol. 63, no. 1, pp. $9-12,2008$.

[82] H.-Y. Lane, C.-H. Lin, Y.-J. Huang, C.-H. Liao, Y.-C. Chang, and G. E. Tsai, "A randomized, double-blind, placebo-controlled comparison study of sarcosine ( $\mathrm{N}$-methylglycine) and d-serine add-on treatment for schizophrenia," International Journal of Neuropsychopharmacology, vol. 13, no. 4, pp. 451-460, 2010.

[83] Y. Hirayasu, S. Sato, H. Takahashi et al., "A double-blind randomized study assessing safety and efficacy following oneyear adjunctive treatment with bitopertin, a glycine reuptake inhibitor, in Japanese patients with schizophrenia," BMC Psychiatry, vol. 16, article no. 66, 2016.

[84] T. Papouin, L. Ladépêche, J. Ruel et al., "Synaptic and extrasynaptic NMDA receptors are gated by different endogenous coagonists," Cell, vol. 150, no. 3, pp. 633-646, 2012.

[85] T.-A. Matsui, M. Sekiguchi, A. Hashimoto, U. Tomita, T. Nishikawa, and K. Wada, "Functional comparison of D-serine and glycine in rodents: the effect on cloned NMDA receptors and the extracellular concentration," Journal of Neurochemistry, vol. 65, no. 1, pp. 454-458, 1995.

[86] D. Quartermain, J. Mower, M. F. Rafferty, R. L. Herting, and T. H. Lanthorn, "Acute but not chronic activation of the NMDAcoupled glycine receptor with D-cycloserine facilitates learning and retention," European Journal of Pharmacology, vol. 257, no. 1-2, pp. 7-12, 1994.

[87] J. Sasabe, T. Chiba, M. Yamada et al., "D-Serine is a key determinant of glutamate toxicity in amyotrophic lateral sclerosis," EMBO Journal, vol. 26, no. 18, pp. 4149-4159, 2007.

[88] U. Heresco-Levy, M. Ermilov, P. Lichtenberg, G. Bar, and D. C. Javitt, "High-dose glycine added to olanzapine and risperidone for the treatment of schizophrenia," Biological Psychiatry, vol. 55, no. 2, pp. 165-171, 2004.

[89] F. A. Carone, S. Nakamura, and B. Goldman, "Urinary loss of glucose, phosphate, and protein by diffusion into proximal straight tubules injured by D-serine and maleic acid," Laboratory Investigation, vol. 52, no. 6, pp. 605-610, 1985.

[90] R. E. Williams, H. Major, E. A. Lock, E. M. Lenz, and I. D. Wilson, "D-Serine-induced nephrotoxicity: a HPLC-TOF/MSbased metabonomics approach," Toxicology, vol. 207, no. 2, pp. 179-190, 2005.

[91] H. Wolosker, E. Dumin, L. Balan, and V. N. Foltyn, "Damino acids in the brain: $\mathrm{D}$-serine in neurotransmission and neurodegeneration," FEBS Journal, vol. 275, no. 14, pp. 35143526, 2008.

[92] Treating Alcohol and Drug Problems in Psychotherapy Practice Doing What Works, Guilford Publications, New York, NY, USA, 2011.

[93] A. Christopoulos, "Allosteric binding sites on cell-surface receptors: novel targets for drug discovery," Nature Reviews Drug Discovery, vol. 1, no. 3, pp. 198-210, 2002.
[94] D. T. Monaghan, M. W. Irvine, B. M. Costa, G. Fang, and D. E. Jane, "Pharmacological modulation of NMDA receptor activity and the advent of negative and positive allosteric modulators," Neurochemistry International, vol. 61, no. 4, pp. 581-592, 2012.

[95] G. L. Collingridge, A. Volianskis, N. Bannister et al., "The NMDA receptor as a target for cognitive enhancement," Neuropharmacology, vol. 64, pp. 13-26, 2013.

[96] B. M. Costa, M. W. Irvine, G. Fang et al., "A novel family of negative and positive allosteric modulators of NMDA receptors," Journal of Pharmacology and Experimental Therapeutics, vol. 335, no. 3, pp. 614-621, 2010.

[97] M. W. Irvine, B. M. Costa, A. Volianskis et al., "Coumarin3-carboxylic acid derivatives as potentiators and inhibitors of recombinant and native N-methyl-d-aspartate receptors," Neurochemistry International, vol. 61, no. 4, pp. 593-600, 2012.

[98] P. Mullasseril, K. B. Hansen, K. M. Vance et al., "A subunitselective potentiator of NR2C- and NR2D-containing NMDA receptors," Nature Communications, vol. 1, article 90, 2010.

[99] X. Zhang, Z.-J. Feng, and K. Chergui, "Allosteric modulation of GluN2C/GluN2D-containing NMDA receptors bidirectionally modulates dopamine release: implication for Parkinson's disease," British Journal of Pharmacology, vol. 171, no. 16, pp. 3938$3945,2014$.

[100] J.-P. Zhao and M. Constantine-Paton, "NR2A-/- mice lack long-term potentiation but retain NMDA receptor and L-type $\mathrm{Ca} 2+$ channel-dependent long-term depression in the juvenile superior colliculus," Journal of Neuroscience, vol. 27, no. 50, pp. 13649-13654, 2007.

[101] L. Liu, T. P. Wong, M. F. Pozza et al., "Role of NMDA receptor subtypes in governing the direction of hippocampal synaptic plasticity," Science, vol. 304, no. 5673, pp. 1021-1024, 2004.

[102] Y. Liu, P. W. Tak, M. Aarts et al., "NMDA receptor subunits have differential roles in mediating excitotoxic neuronal death both in vitro and in vivo," Journal of Neuroscience, vol. 27, no. 11, pp. 2846-2857, 2007.

[103] M. Chen, T.-J. Lu, X.-J. Chen et al., "Differential roles of NMDA receptor subtypes in ischemic neuronal cell death and ischemic tolerance," Stroke, vol. 39, no. 11, pp. 3042-3048, 2008.

[104] Y. Sun, X. Cheng, L. Zhang et al., "The functional and molecular properties, physiological functions, and pathophysiological roles of GluN2A in the central nervous system," Molecular Neurobiology, pp. 1-14, 2016.

[105] T. E. Bartlett, N. J. Bannister, V. J. Collett et al., "Differential roles of NR2A and NR2B-containing NMDA receptors in LTP and LTD in the CA1 region of two-week old rat hippocampus," Neuropharmacology, vol. 52, no. 1, pp. 60-70, 2007.

[106] H. Monyer, N. Burnashev, D. J. Laurie, B. Sakmann, and P. H. Seeburg, "Developmental and regional expression in the rat brain and functional properties of four NMDA receptors," Neuron, vol. 12, no. 3, pp. 529-540, 1994.

[107] C. Rauner and G. Köhr, "Triheteromeric NR1/NR2A/NR2B receptors constitute the major N-methyl-D-aspartate receptor population in adult hippocampal synapses," The Journal of Biological Chemistry, vol. 286, no. 9, pp. 7558-7566, 2011.

[108] S. Savanthrapadian, A. R. Wolff, B. J. Logan, M. J. Eckert, D. K. Bilkey, and W. C. Abraham, "Enhanced hippocampal neuronal excitability and LTP persistence associated with reduced behavioral flexibility in the maternal immune activation model of schizophrenia," Hippocampus, vol. 23, no. 12, pp. 1395-1409, 2013. 
[109] M. Volgraf, B. D. Sellers, Y. Jiang et al., "Discovery of GluN2A-selective NMDA receptor Positive Allosteric Modulators (PAMs): tuning deactivation kinetics via structure-based design," Journal of Medicinal Chemistry, vol. 59, no. 6, pp. 27602779, 2016.

[110] G. E. Hardingham and H. Bading, "Synaptic versus extrasynaptic NMDA receptor signalling: implications for neurodegenerative disorders," Nature Reviews Neuroscience, vol. 11, no. 10, pp. 682-696, 2010.

[111] A. M. Kaufman, A. J. Milnerwood, M. D. Sepers et al., "Opposing roles of synaptic and extrasynaptic NMDA receptor signaling in cocultured striatal and cortical neurons," Journal of Neuroscience, vol. 32, no. 12, pp. 3992-4003, 2012.

[112] G. Maccaferri and R. Dingledine, "Control of feedforward dendritic inhibition by NMDA receptor-dependent spike timing in hippocampal interneurons," The Journal of Neuroscience, vol. 22, no. 13, pp. 5462-5472, 2002.

[113] G. Nyíri, F. A. Stephenson, T. F. Freund, and P. Somogyi, "Large variability in synaptic N-methyl-D-aspartate receptor density on interneurons and a comparison with pyramidal-cell spines in the rat hippocampus," Neuroscience, vol. 119, no. 2, pp. 347363, 2003.

[114] J. R. P. Geiger, J. Lübke, A. Roth, M. Frotscher, and P. Jonas, "Submillisecond AMPA receptor-mediated signaling at a principal neuron-interneuron synapse," Neuron, vol. 18, no. 6, pp. 1009-1023, 1997.

[115] M. C. Angulo, J. Rossier, and E. Audinat, "Postsynaptic glutamate receptors and integrative properties of fast- spiking interneurons in the rat neocortex," Journal of Neurophysiology, vol. 82, no. 3, pp. 1295-1302, 1999.

[116] H.-X. Wang and W.-J. Gao, "Cell type-specific development of NMDA receptors in the interneurons of rat prefrontal cortex," Neuropsychopharmacology, vol. 34, no. 8, pp. 2028-2040, 2009. 

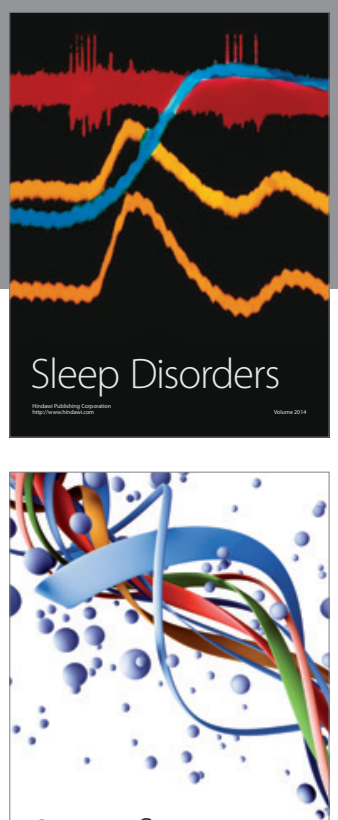

Scientifica
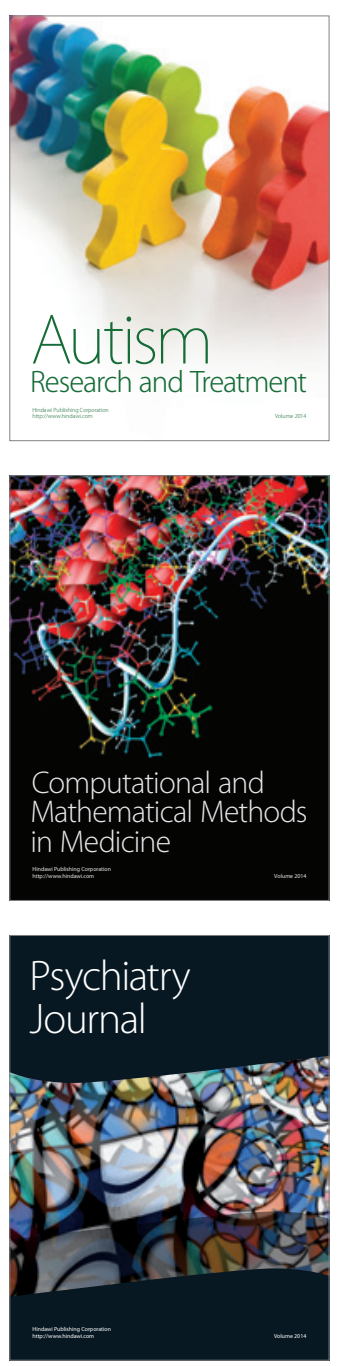
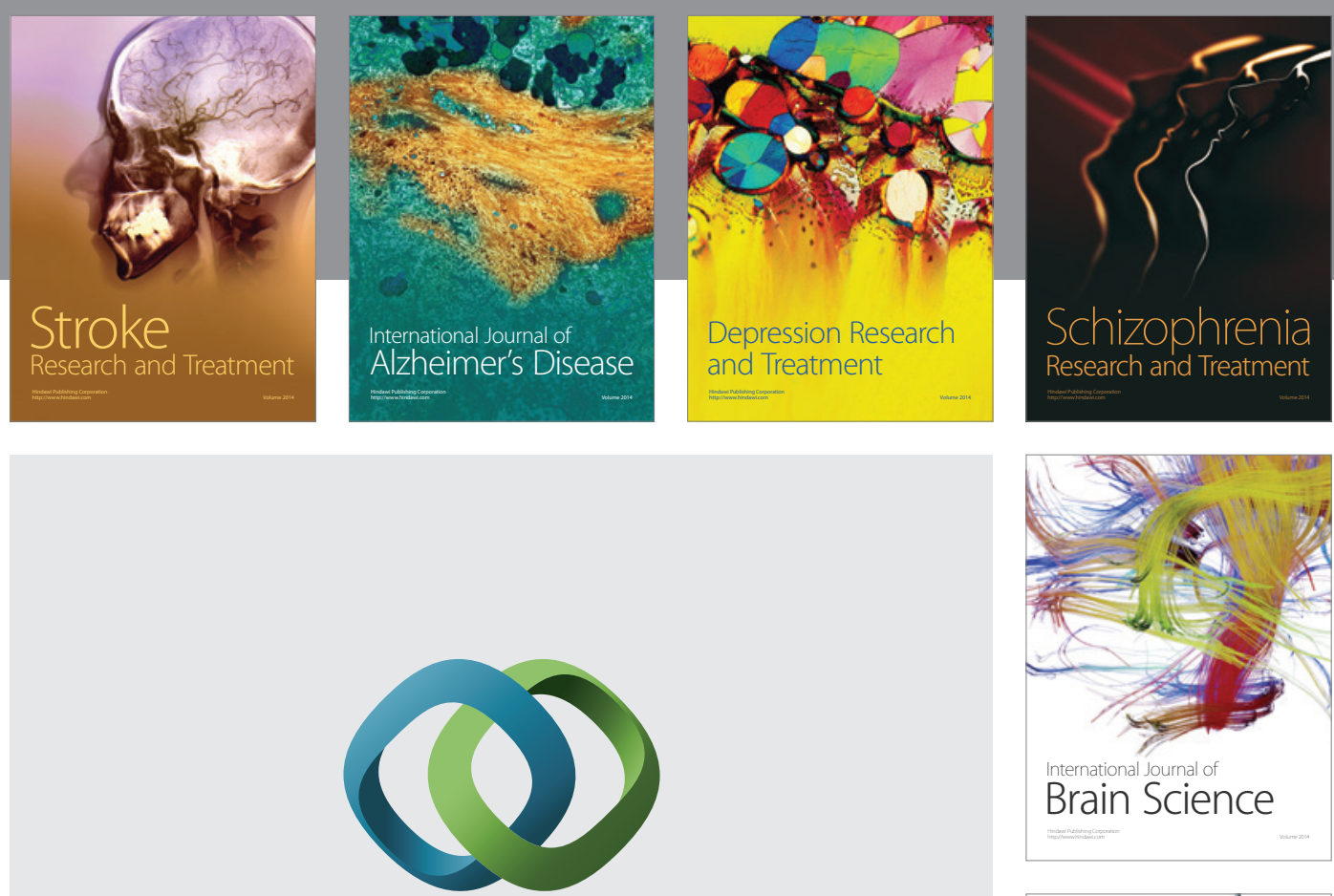

\section{Hindawi}

Submit your manuscripts at

https://www.hindawi.com
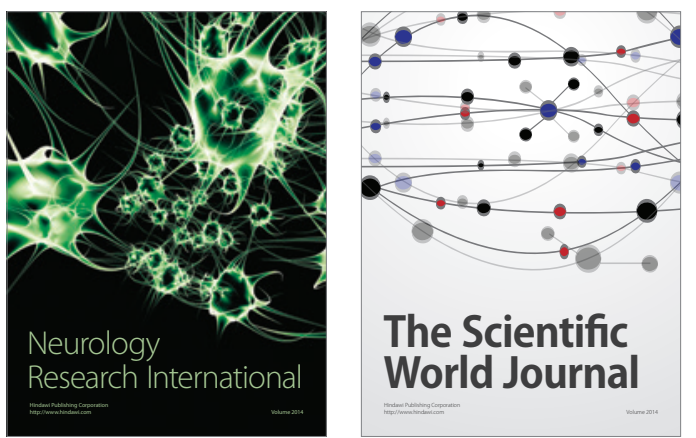

The Scientific World Journal

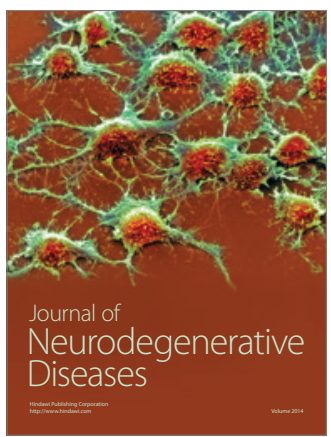

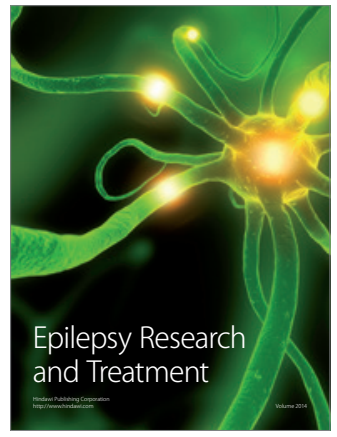

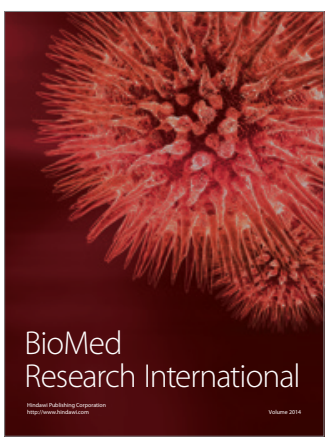

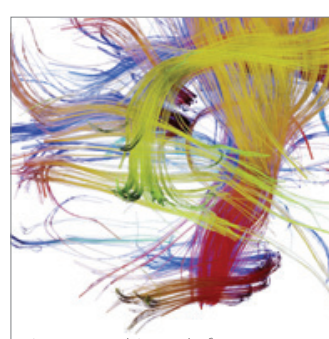

Brain Science

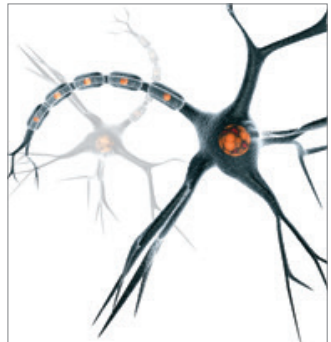

Neural Plasticity
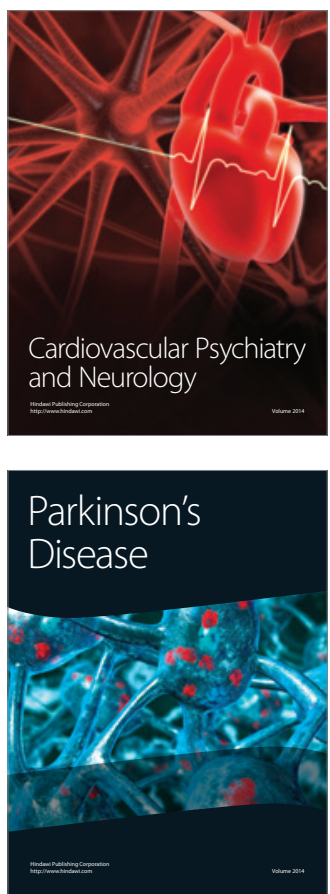